\title{
Written communication for itu discharges: a quality improvement project
}

\author{
D Bendel', H Gardner, M Outram \\ From ESICM LIVES 2015 \\ Berlin, Germany. 3-7 October 2015
}

\section{Introduction}

ITU patients are complex, and detailed written handover in the form of a discharge letter plays a central role in patient safety [1]. We sought to characterise and improve upon the quality of our written handover on discharge.

\section{Objectives}

To improve the quality of written discharge communication.

\section{Methods}

In 2014, we audited our discharge summaries over a two month period. Our adopted standards were the National
Institute of Health and Clinical Excellence (NICE) clinical guidelines 50 and 83 [2],[3]. They detail various domains that should be present on a summary. These domains formed the basis of our audit proforma.

Discharge summaries were then scored according to whether the domains were satisfied or not. The total score for each domain was then divided by the total number of discharge summaries, in order to indicate the percentage of summaries that satisfied each domain.

Following this audit, we introduced an electronic discharge summary template. The template is composed of mandatory headings that cannot be deleted. Each heading addresses a different domain from NICE guidance, and is

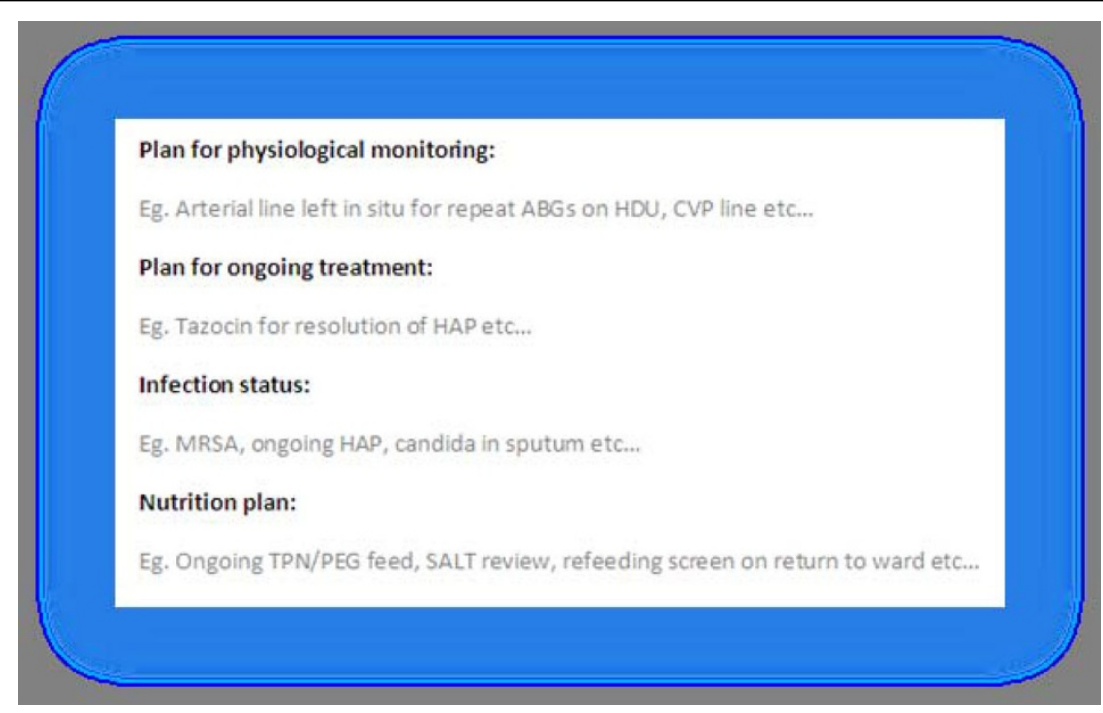

Figure 1 Detail of the new discharge summary. 


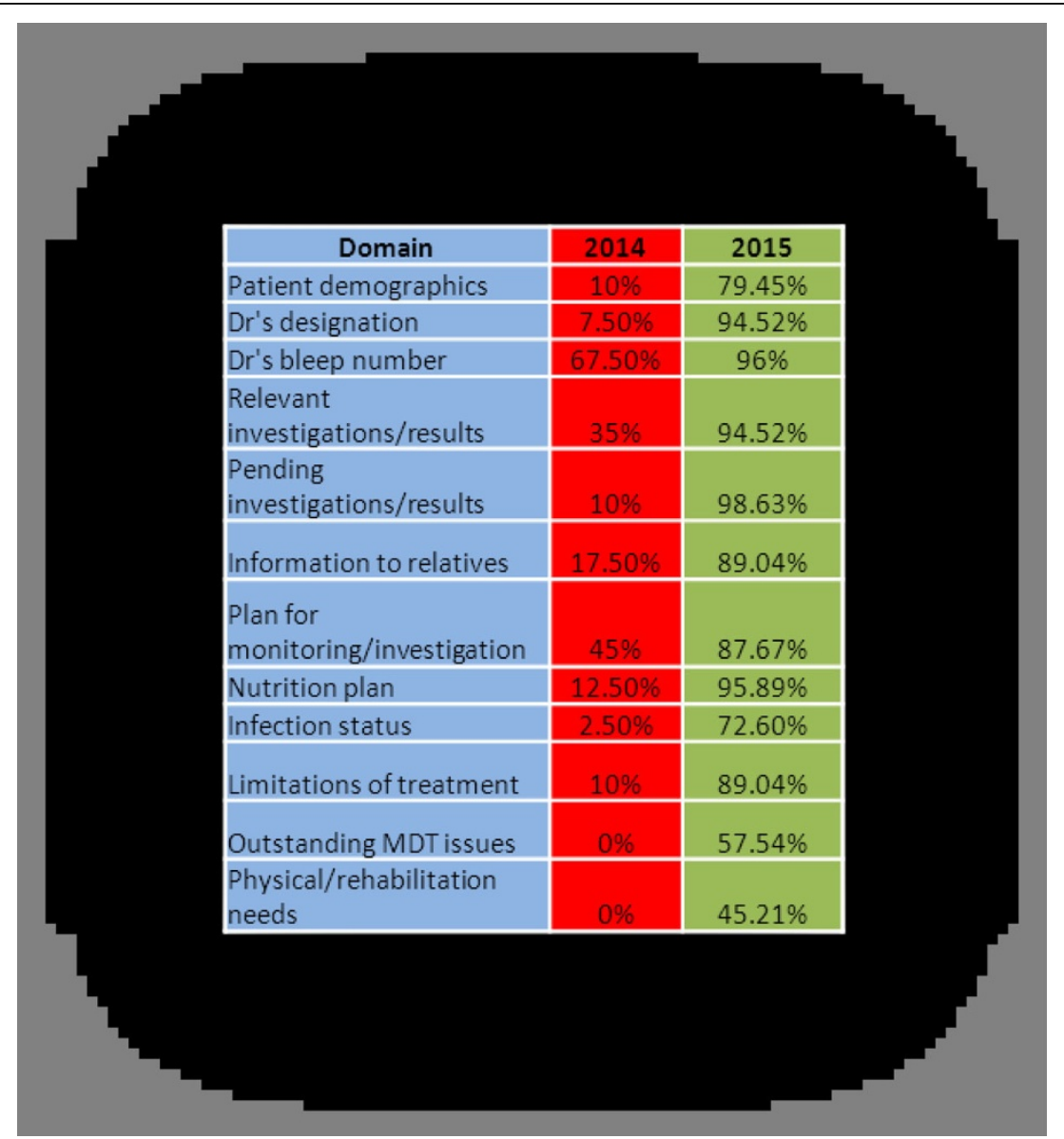

Figure 2 Areas of significant improvement

followed by a free-text box. They contain suggestions in grey that disappear upon entering information (Figure 1).

We closed the audit loop in 2015.

\section{Results}

Our discharge summaries fell short of our adopted standards across several domains. Frequent omissions included legal aspects such as patient demographics (10\%), Dr's designation (8\%) and bleep number (68\%). Other regularly omitted domains included a plain-English summary for relatives $(18 \%)$, relevant investigations $(35 \%)$ and a plan for patient monitoring on return to the ward (45\%).

This was due, at least in part, to the document's 'freetext' nature.

On re-audit, the legal aspects of our summaries are almost $100 \%$ compliant. There is improvement to areas such as reason for admission (99\%) and diagnosis (85\%). New information that now features on our summaries includes pending investigations, nutrition plan, infection status, limitations of treatment and information for relatives (figure 2).
Our new discharge template has dramatically improved the quality of our written handovers.

\section{Conclusions}

The safe handover of ITU patients relies on detailed written communication, in line with NICE guidance. Simple technological interventions such as the discharge template described here are an effective way of achieving this.

Published: 1 October 2015

\section{References}

1. van Sluisveld N, Hesselink G, van der Hoeven JG, Westert G, Wollersheim H, Zegers M: Improving clinical handover between intensive care unit and general ward professionals at intensive care unit discharge. Intensive Care Medicine 2015, 41(4):589-604.

2. National Institute for Health and Clinical Excellence (NICE): CG50 Acutely ill patients in hospital: NICE guideline. London: NICE; 2007.

3. National Institute for Health and Clinical Excellence (NICE): CG83 Critical Illness Rehabilitation: NICE Guideline. London: National Institute for Health and Clinical Excellence; 2009

doi:10.1186/2197-425X-3-S1-A143

Cite this article as: Bendel et al:: Written communication for itu discharges: a quality improvement project. Intensive Care Medicine Experimental 2015 3(Suppl 1):A143. 